\title{
Approche comparée de méthodes de classification d'images aériennes: une étude de cas
}

\author{
Régis Caloz, Abram Pointet, Lausanne \\ Claude Collet, Fribourg
}

\section{Introduction}

Dans le domaine de la détermination des ressources terrestres jusqu'à une date récente, les images de télédétection étaient davantage d'origine spatiale qu'aérienne. Leur résolution s'étendait de $5 \mathrm{~m}$ à plusieurs dizaines de mètres. Les extractions de catégories d'occupation du sol étaient réalisées (et le sont encore) par des classificateurs non-dirigés (clustering) ou dirigés dont le modèle théorique le plus réussi est fondé sur le maximum de vraisemblance. Les résolutions mentionnées n'autorisant pas de délimiter des unités d'occupation du sol avec une grande précision, l'accent est mis sur la dimension thématique plutôt que géométrique.

Les nouvelles images satellitales à très haute résolution $(<5 \mathrm{~m})$ disposent de propriétés similaires à celles issues de prises aériennes. De surcroît, pour bénéficier des traitements par outils numériques, les photos aériennes sont de plus en plus numérisées, de sorte qu'aujourd'hui la différence entre un produit spatial et un produit aérien s'est estompée. Ainsi, les images aériennes scannées et satellitales se traitent de manière similaire, bien que leurs informations spectrales soient de nature différente, l'image aérienne étant produite par scannage d'une composition colorée.

La très haute résolution spatiale a fait apparaître davantage d'exigences sur la précision géométrique des résultats mettant ainsi la dimension spatiale au même rang que la dimension spectrale. Les classificateurs conventionnels basés sur la seule dimension spectrale se sont avérés inappropriés dans de nombreux cas. Dès la fin de la décennie 70, des auteurs (NAGAO \& Matsuyama 1979; Haralick \& Shapiro 1985) avaient proposé une approche exploitant simultanément les deux dimensions spatiale et spectrale. Les classificateurs exploitant cette condition sont dits contextuels ou de croissance de région. A notre connaissance, le premier logiciel commercial basé sur ces concepts est eCognition de la firme Definiens, dont la première version est apparue en 1997.

Nous avons choisi d'analyser et de mettre en évidence le comportement de cette dernière approche par un cas d'étude basé sur la détermination des dégâts forestiers produits par l'ouragan Lothar, le 26 décembre
1999. Notre objectif n'est donc pas de produire une cartographie des dégâts par une procédure numérique susceptible de se substituer à celle réalisée par photointerprétation, mais de mettre en parallèle les deux démarches, d'en dégager et de formuler leurs propriétés respectives. ScHWARz et al. (2002) ont également exploité le même logiciel pour la détermination de dégâts forestiers, mais à partir des images SPOT et Ikonos.

Le choix de l'étude de cas est motivé par les difficultés probables que l'on affronte pour discriminer les différents états d'ouverture de la forêt: ceux produits par l'ouragan à divers degrés, ceux correspondant à des clairières créées par l'homme.

Notre démarche est subdivisée en trois parties. Dans la première, nous présentons brièvement, à l'intention du lecteur non spécialisé, en traitement d'image, les principes de la classification par croissance de région. Puis, nous procédons successivement à une classification de l'image par une approche non-dirigée (clustering) et par une méthode de croissance de région offerte par le logiciel eCognition (2001).

\section{Approche par croissance de région}

\subsection{Principes de la procédure par croissance de région}

La procédure par croissance de région est de nature séquentielle. On la divise en deux phases principales : les agrégations de bas et de haut niveaux.

La première étape consiste, à partir de pixels définis comme germes, à former de petites régions de taille limitée par un seuil donné, considérées comme homogènes du point de vue spectral, de quelques dizaines à quelques centaines de pixels selon le degré d'hétérogénéité de l'image (cf. l'image 5a).

Lors de la phase suivante, agrégation de haut niveau, l'extension des régions se poursuit mais avec un choix beaucoup plus large de critères, tout en maintenant la contrainte d'adjacence: tout indicateur statistique susceptible d'être établi sur les régions initiales, indices spectraux, indices de texture, indices de forme et le maximum de vraisemblance. Les agrégations sont interrompues lorsque l'analyste juge que la segmentation de l'image obtenue lui permet d'associer les classes spectrales aux catégories thématiques désirées. 


\begin{tabular}{|l|l|}
\hline Phase & Fonctions \\
\hline 1. Sélection des germes & $\begin{array}{l}\text { Fenêtre glissante sans } \\
\text { chevauchement } \\
\text { Semis aléatoire } \\
\text { «bassin versant » } \\
\text { Autres }\end{array}$ \\
\hline $\begin{array}{l}\text { 2. Agrégation de bas niveau - Création de } \\
\text { petites régions }\end{array}$ & $\begin{array}{l}\text { Distance spectrale } \\
\text { Différentiel d'indice }\end{array}$ \\
$\begin{array}{l}\text { Agrégation de pixels adjacents sous la double } \\
\text { lendition de seuils fixés respectivement pour } \\
\text { Résultats: fragmentation de l'image en petites } \\
\text { régions }\end{array}$ & $\begin{array}{l}\text { Structure markovienne } \\
\text { Seuillages d'histogramme }\end{array}$ \\
& Extraction de contours \\
Segmentation en polygones de \\
3. Agrégation de haut niveau \\
$\begin{array}{l}\text { Agrégation de régions adjacentes présentant } \\
\text { une similitude en référence à un paramètre } \\
\text { fixé }\end{array}$ & Voronoï \\
& Segmentation en tétrarbre \\
\hline
\end{tabular}

Tableau 1: Processus de la croissance de région par approche ascendante Regional growth classification approach using ascending procedure «Regional growth»-Klassifikation mit der Nächstgelegener-Nachbar-Methode

L'approche décrite, dénommée également contextuelle, présente un grand avantage dans la mesure où il n'est pas nécessaire de définir a priori les relations entre classes spectrales et classes thématiques. Le processus d'agrégation est contrôlé à chaque étape, l'analyste dispose donc de moyens de le faire évoluer dans le sens le plus conforme aux objectifs. Des classes sans intérêts sont regroupées; pour d'autres, au contraire, les critères d'agrégation sont fixés pour conserver un maximum de capacité discriminante.

La détermination des germes est évidemment une étape importante du processus. La littérature présente à ce propos de nombreuses manières de le faire notamment (Ahuja \& Schachte 1985; Chassery \& Melkemi 1991; Yu \& Wang 1999; HoJjatoleslami \& KitTleR 1998; INAMPUDI \& NMURALIKRISHNA 1999). La plus fréquemment citée est probablement celle dite de bassin versant de Vincent \& Sollle (1991). Les germes sont les pixels de plus faible luminance, ils sont assimilés à des exutoires de bassins versants. L'exutoire définit le bassin versant, le germe donne naissance à la région! L'image sur laquelle s'appliquent les traitements peut être l'image originale, une image en composantes principales, l'image gradient dérivée des précédentes par un filtre Sobel. Le tableau 1 présente une synthèse des principales phases et fonctions intervenant dans un processus basé sur une approche par région (CocQUEREZ \& PHILIPP 1995).

Les processus de classification diffèrent dans leur principe d'agrégation des pixels ainsi que par la phase du traitement pendant lequel est injectée l'information thématique. Dans une procédure non-dirigée, l'analyste intervient à deux reprises, lorsque qu'il fixe le nombre de classes et lorsqu'il affecte les classes spectrales aux catégories thématiques. Pour le cas dirigé, l'information thématique est injectée par les signatures spectrales. Le cas de croissance de région est différent. Ce processus offrant plus de choix, l'analyste a la possibilité d'apporter l'information thématique à plusieurs reprises: lors de l'agrégation à bas niveau par le choix de la taille des régions, lors des phases suivantes par la sélection des paramètres exploités. Ces derniers constituent la signature thématique des régions. Les diagrammes, accompagnant les deux approches 


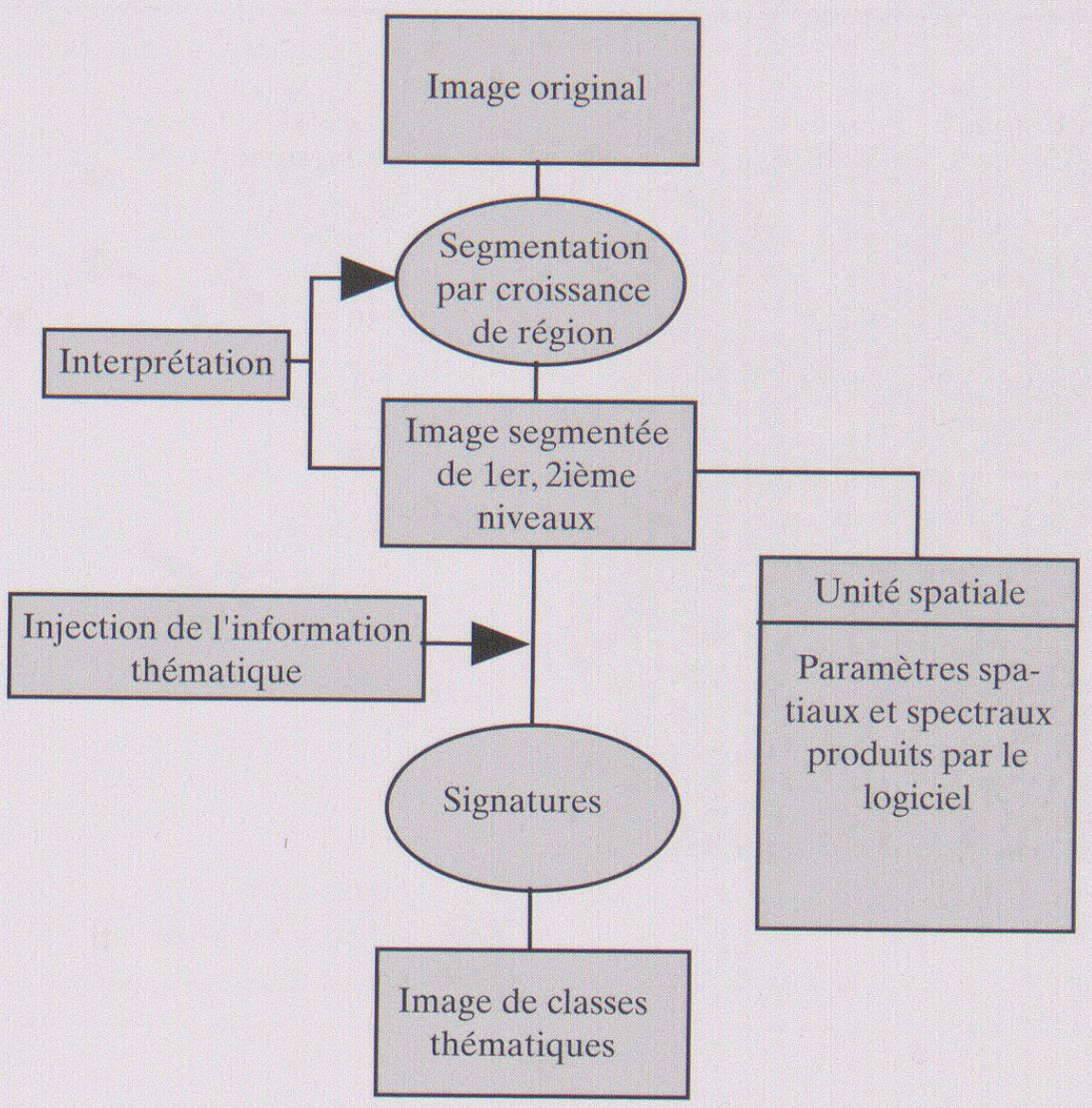

Figure 1: Diagramme simplifié des traitements d'un processus par croissance de région Simplified flowchart for a regional growth classification procedure Vereinfachtes Diagramm mit den Rechenschritten der «regional growth»-Klassifikation (schéma conçu par l'auteur)

présentées dans les sections suivantes, nous indiquent à quelle séquence est injectée l'information spatiale (figures 1 et 4 ).

\section{Cas d'étude : détermination de dégâts forestiers dûs à l'ouragan Lothar}

\subsection{Site d'étude}

Le région choisie pour l'étude est située au sud du canton de Fribourg aux coordonnées centrales (57’000 x 177’000) de la carte nationale suisse. La principale agglomération est le village de Posat. La forêt a subi d'importants dégâts lors du passage de l'Ouragan Lothar en décembre 1999. Pour évaluer l'ampleur des destructions, les services cantonaux et fédéraux ont procédé à un levé par photointerprétation d'images aériennes spécialement acquises à cet effet. Notons que les météorologues utilisent le mot ouragan dès qu'une tempête connaît des vents soufflant à plus de $128 \mathrm{~km} / \mathrm{hres}$, vitesse largement dépassée par Lothar pour lequel des vitesses extrêmes de $240 \mathrm{~km} / \mathrm{hres}$ ont été observées.

\subsubsection{Caractéristiques de la photointerprétation}

Les clés d'interprétation ont été fixées par l'Office fédéral de l'Environnement, de la Forêt et du Paysage (OFEFP). On considère les trois catégories suivantes : Catégorie 1: Dégât total, plus du $80 \%$ de la surface forestière est détruite

Catégorie 2: Dégâts diffus: entre $40 \%$ et $80 \%$ de la surface

Catégorie 3: Îlots sans dégâts au sein d'une surface de dégâts.

On ne prend pas en compte des surfaces touchées inférieures à 1 ha.

La figure 2 présente les résultats de la photointerprétation réalisée par le bureau d'ingénieurs Scherrer AG, 9650 Nesslau.

\subsubsection{Caractéristiques de l'image}

Les informations à référence spatiale, exploitées dans cette approche de zonage par classification conventionnelle, sont d'une part une composition colorée numérique de l'orthophotographie à une résolution 
a) Orthoimage «Posat»

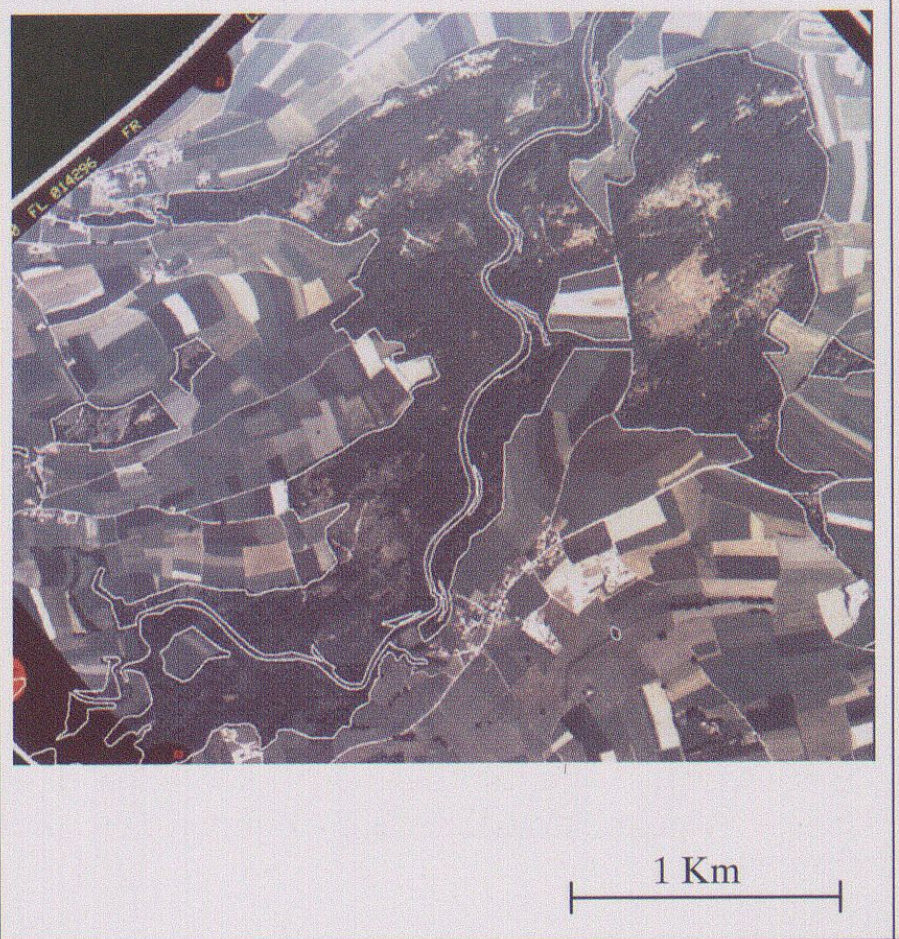

b) Cartographie des dégâts forestiers issue de la photo-interprétation

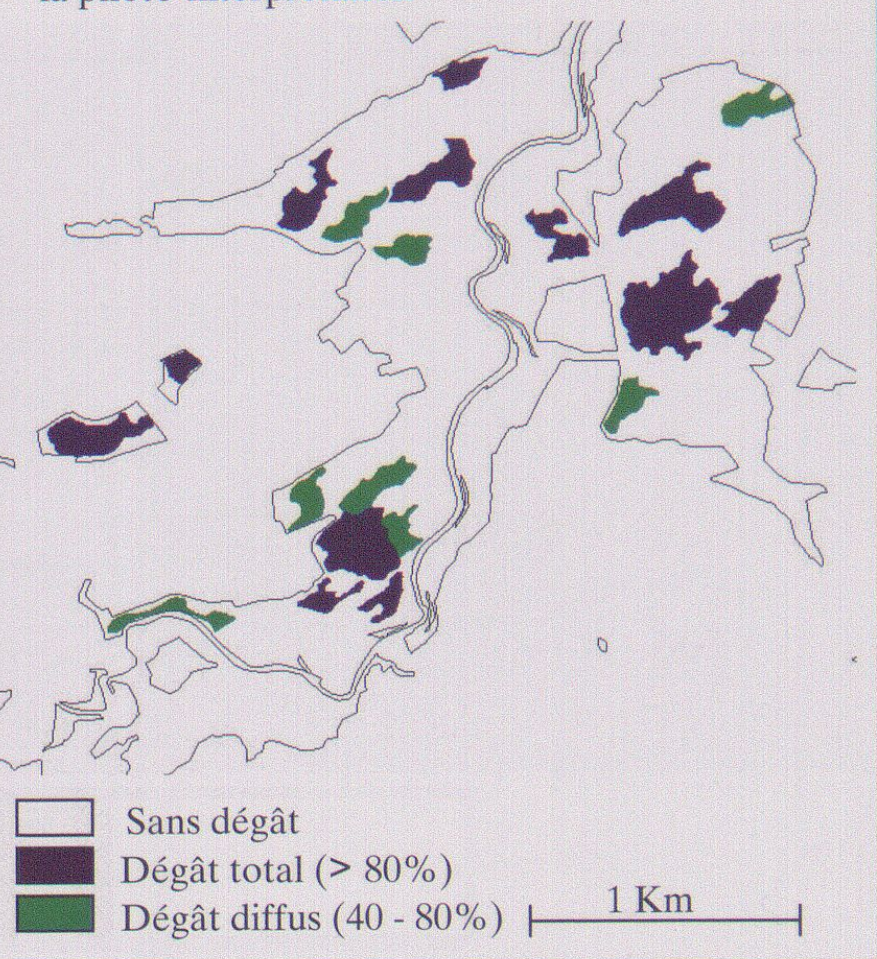

Figure 2: Image de la forêt Posat et cartographie des dégâts Orthophoto and map of forest damages in the study area of Posat Orthophoto und Waldschadenkarte der Region Posat Source: Service forestier du canton de Fribourg

d'environ $70 \mathrm{~cm}$ et d'autre part les fichiers vecteur délimitant les zones de forêt (Vecteur 25 de l'OFT), ainsi que les zones de classes de dégâts, telles qu'elles sont définies par photointerprétation (figure $2 b$ ). Les statistiques de l'image sont présentées sur le tableau 2.

Dans un souci d'orienter la classification sur les valeurs spectrales spécifiques à ces zones forestières, une extraction par masquage a été réalisée sur ces der-

\begin{tabular}{|l|r|r|r|}
\hline & Bleu & Vert & Rouge \\
\hline Minimum & 34 & 17 & 32 \\
\hline Maximum & 120 & 160 & 155 \\
\hline Moyenne & 74 & 62 & 59 \\
\hline
\end{tabular}

Tableau 2: Statistique de l'image aérienne utilisée Aerial image statistics Statistische Auswertungen des Luftbildes nières. C'est sur cette image masquée que seront appliquées les procédures de classification.

\subsection{Zonage par classification conventionnelle}

L'examen visuel de l'image de Posat dévoile clairement qu'il est possible d'interpréter, au niveau de l'unité d'observation qu'est le pixel, la teinte des couleurs en termes d'importance du couvert forestier. Les teintes très claires correspondent à des zones dégradées et celles plus foncées expriment la présence de végétal. On y observe cependant une diminution régulière prononcée de la luminosité des teintes en direction du sud, artefact fréquent (hot spot) et contraignant dans ce type de document.

\subsubsection{Démarche des expérimentations}

Parmi les types de classificateurs conventionnels, c'est celui des non-dirigés qui a été choisi. Ce choix se justifie par la nature même de la thématique à extraire. Nous souhaitons identifier des zones de dégâts forestiers qui s'expriment, au niveau du pixel, par une faible présence ou même une absence totale de matériel végétal vert. Cette approche permet donc de mettre en évidence le potentiel discriminatoire des valeurs spec- 


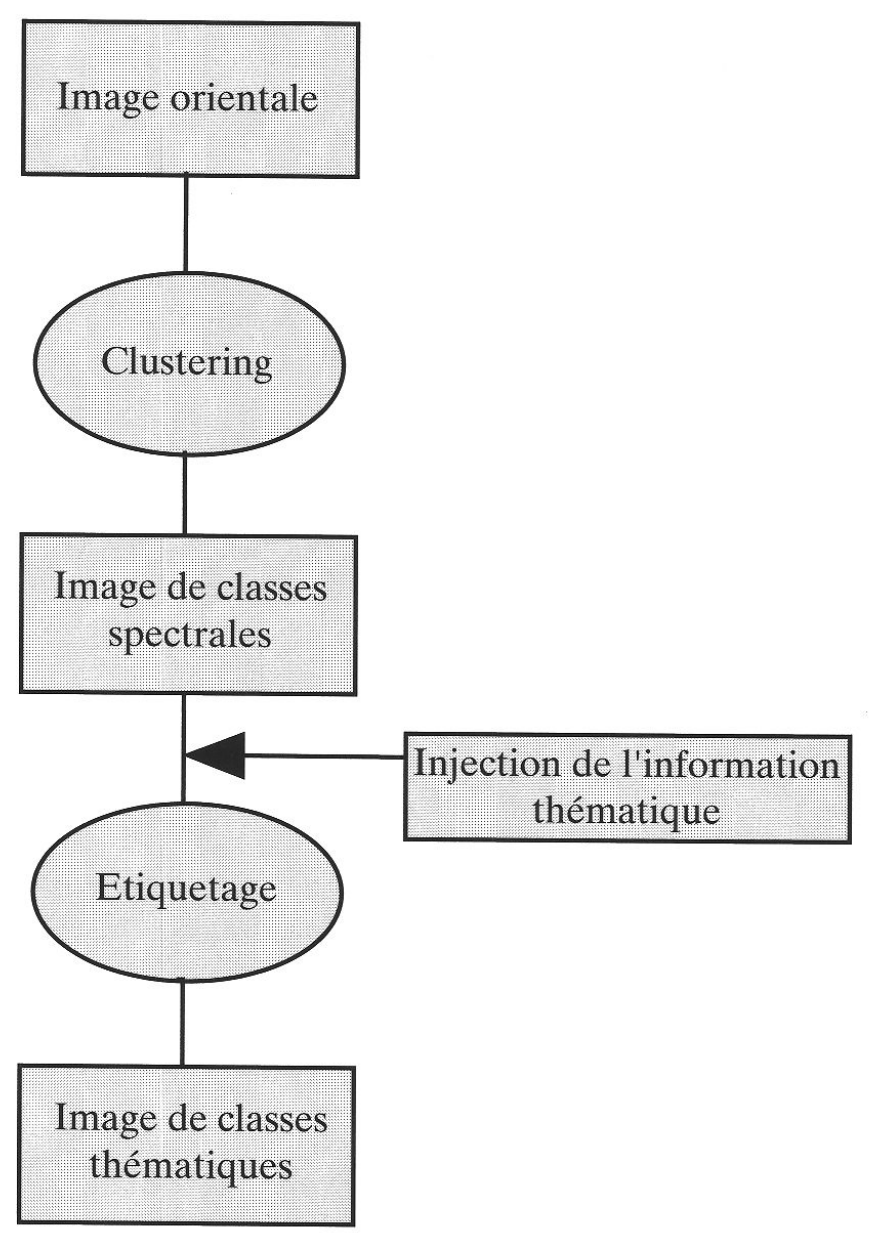

Figure 3: Démarche générale de classification non dirigée (Clustering)

Flowchart of unsupervised classification method (clustering)

Vorgehenswise bei der unüberwachten Klassifikation (Clustering)

(schéma conçu par l'auteur)

trales dans les zones forestières. La figure 2 présente les étapes du processus.

Les procédures de classification conventionnelle limitent leur champ d'action à la dimension thématique de l'information disponible. Les propriétés telles que l'arrangement spatial des unités d'observation sont ignorées durant le processus de classification. Notre objectif étant de produire des régions ou zones de forêt dégradée et de les mettre en relation avec les objets produits par photointerprétation, il sera nécessaire de prolonger la démarche de classification illustrée par la figure 3 par une étape de prise en compte des propriétés spatiales des ensembles de pixels classifiés.

La confrontation des résultats de classification avec ceux de la photointerprétation n'est pas à considérer comme une validation au sens strict du terme. Elle a avant tout valeur d'évaluation du potentiel d'une telle approche automatisée. L'interprétation visuelle procède par une délimitation de zones d'une certaine taille ( $>1 \mathrm{ha}$ ) contenant des dégâts forestiers, suivie d'une appréciation de l'importance en termes d'étendue de ces dégâts. De façon différente, l'approche de classification tend à identifier des classes ou des catégories de dégâts au niveau de l'unité d'observation qu'est le pixel. Si les deux approches se justifient, leur résultat ne répondra pas exactement à la même problématique d'investigation.

La première tentative de classification s'est soldée par des résultats peu convaincants qui ne sont pas présentés ici. Les classes spectrales produites, quel que soit leur nombre, exprimaient bien des degrés de dégradation du couvert forestier, mais posaient deux problèmes majeurs: l'un relatif à l'hétérogénéité spatiale dans la distribution des classes, l'autre à l'hétérogénéité du contenu des classes selon leur localisation dans l'image. Le premier problème a pour origine la résolution même de l'unité d'observation qui est de $70 \mathrm{~cm}$ environ. À cette échelle de mesure du phénomène, la variabilité des valeurs spectrales est très grande car les unités d'observation correspondent à des éléments trop fins des objets d'intérêt qui composent la forêt. Il sera nécessaire d'agréger ces unités d'observation originales en pixels de résolution moindre, rendant mieux compte de l'état de la canopée. Le second problème a pour origine la décroissance systématique de la luminosité de l'image en direction du sud. Cet effet mentionné précédemment ne peut être corrigé par des traitements simples et de façon globale pour l'ensemble des photographies numériques couvrant une vaste région d'étude. Sa correction hypothéquerait ainsi de manière trop importante le potentiel opérationnel d'une démarche de classification numérique. Nous avons donc choisi de conserver ce biais systématique de luminosité dans l'image traitée lors de la seconde expérimentation.

Lors de cette seconde tentative, la résolution de l'image originelle a été réduite par agrégation à une taille de 2 mètres environ. La démarche suivie durant cette expérimentation s'articule selon les étapes suivantes :

1. Classification non dirigée de l'image. La procédure produisant 7 catégories spectrales a été retenue.

2. Etiquetage thématique des classes spectrales. Ces classes expriment principalement des degrés de couverture végétale dans les pixels. Afin de mettre en évidence les zones dégradées et pour compenser les effets du biais dans la luminosité, ces 7 classes ont été regroupées et étiquetées en 5 classes thématiques.

3. Extraction de la catégorie de forêt dégradée. À cette étape de l'analyse, les trois niveaux de dégradation sont fusionnés en une seule catégorie, afin d'identifier des régions ou zones de dégradation. 
4. Lissage des zones dégradées. L'homogénéisation des zones dégradées et de leur contour a été réalisée par l'application d'une procédure de fermeture morphologique avec une fenêtre contextuelle de 3x3 (CAloz \& Collet 2001: 185).

5. Classement des superficies des zones dégradées. Les zones retenues ont été identifiées et leur superficie a été estimée en ha. Le classement de ces aires permet de distinguer l'étendue de la dégradation et ainsi d'opérer un filtrage ultérieur afin d'éliminer, si cela paraît raisonnable, les zones de petite taille dont la dégradation pourrait avoir une autre origine que la tempête.

\subsubsection{Résultats et commentaires des différentes étapes}

Voir figure 4: Résultats et commentaires des différentes étapes de la démarche de classification non dirigée. Comparaison avec les résultats obtenus par photointerprétation.

\subsubsection{Conclusions sur cette approche}

La démarche présentée pour l'identification des zones dégradées illustre de manière convaincante l'importance de l'intervention de l'analyste dans une approche qui pourtant fait appel à un type de classificateur «non dirigé».

La confrontation des résultats produits avec ceux de la photointerprétation est riche d'enseignement à différents niveaux. Comme on l'a indiqué précédemment, la nature des objets à produire diverge entre ces deux approches. Toutefois, on observe des similitudes dans la définition de la plupart des zones de «dégât total» (>80\%) et de celles identifiées comme zones de «dégât diffus» $(40-80 \%)$ qui contiennent une certaine proportion de régions dégradées. Les dissemblances sont de deux types. D'une part, des régions dégradées relativement vastes n'ont pas été retenues au niveau de l'interprétation visuelle; est-ce une omission ou avaientelles une autre origine que l'ouragan dévastateur? D'autre part, les quelques zones de dégâts situées dans la partie sud de l'image ont été systématiquement sous-estimées par cette démarche de classification. On en a identifié la cause précédemment et l'une des solutions possibles consisterait à ne traiter que la partie de l'image possédant la plus forte dynamique. Ceci est envisageable dans un contexte de production, étant donné le recouvrement de $75 \%$ de ces séries de photographies aériennes.

\subsection{Zonage par le classificateur contextuel eCognition}

\subsubsection{Principe de fonctionnement d'eCognition}

Le logiciel eCognition exploite une procédure de croissance de région décrite dans la section 2; il offre trois paramètres d'ajustement accessibles à l'analyste. Le premier, nommé Facteur d'échelle contrôle simultanément les seuils d'agrégation fixés pour la distance spectrale et spatiale. La pondération entre les deux variables est interne au logiciel. Le paramètre Couleur permet d'ajuster le poids accordé soit à la dimension spectrale, soit à la forme de la région. Un troisième paramètre, Rugosité - Compacité, contrôle la sinuosité des lignes de contour ainsi que la forme des régions. Par exemple, une rugosité faible favorise les formes plus géométriques.

Le zonage est réalisé par étape. Pour mettre en évidence les évolutions, nous avons choisi de les réaliser en deux phases. Les images successives ainsi que les commentaires sont présentés en parallèle.

L'étiquetage ou l'insertion de l'information thématique intervient lorsque l'analyste juge que les unités définies par le classificateur sont de taille suffisante pour une interprétation thématique et que les frontières sont respectées. L'affectation est réalisée par les algorithmes conventionnels. Dans le cas présent, nous avons appliqué simplement l'algorithme de distance minimale. Notons qu'à ce stade, la signature comprend les paramètres dérivés d'informations spectrales et celles spatiales établies par la création des régions. Cette opération est appliquée à l'état d'agrégation représenté sur l'image $5 \mathrm{~b}$.

\section{Résultats et analyse}

Dans quelle mesure, une procédure numérique peutelle se substituer à la photointerprétation? La question intéresse, sans aucun doute, les services forestiers à la recherche légitime d'une solution efficace et moins coûteuse. Il n'était pas dans le propos de cette communication de procéder à une évaluation complète d'une approche numérique. C'est pourquoi, nous nous limitons à quelques commentaires suscités par la mise en œuvre des deux procédures de classification.

Validation des résultats: Nous n'avons pas procédé à une évaluation des résultats de la classification par les moyens conventionnels, c'est-à-dire la matrice de confusion ainsi que l'indice Kappa. Cette vérification passe par la notion de «vérité sol». Dans le cas présent, cela signifierait qu'une codification rigoureuse des dégâts aurait été proposée tant du point de vue thématique que géométrique. Dans la logique de la photointerprétation, les limites des unités spatiales sont définies de manière approximative comme l'illustre la figure $4 \mathrm{~d}$. Les résultats des classifications présentent quant à eux des limites d'unité très sinueuses reflétant bien ce que l'on observe sur le terrain. Dans ce sens, ils sont plus proches de la réalité. Cependant, pour le gestionnaire de la forêt, cette résolution peut 


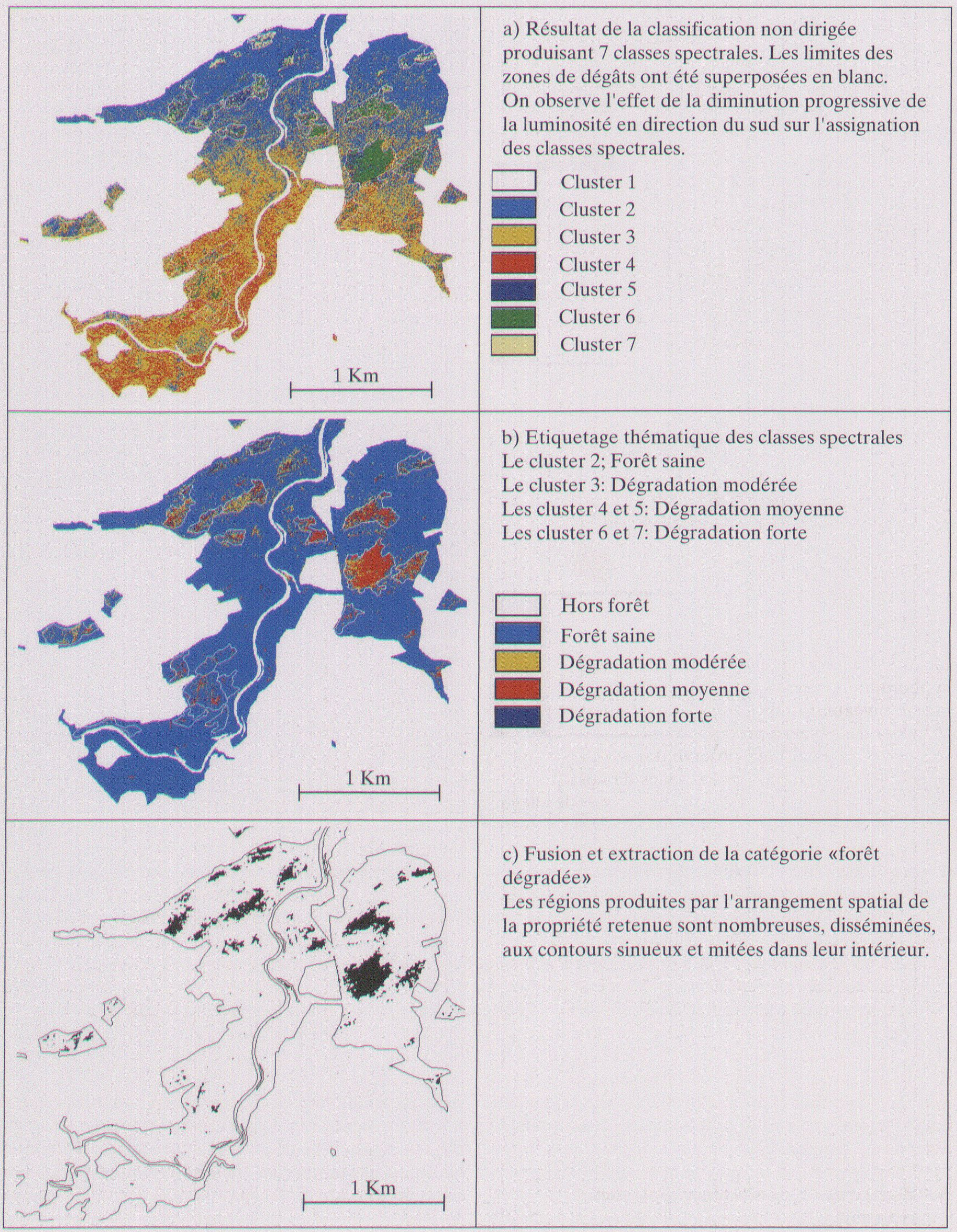




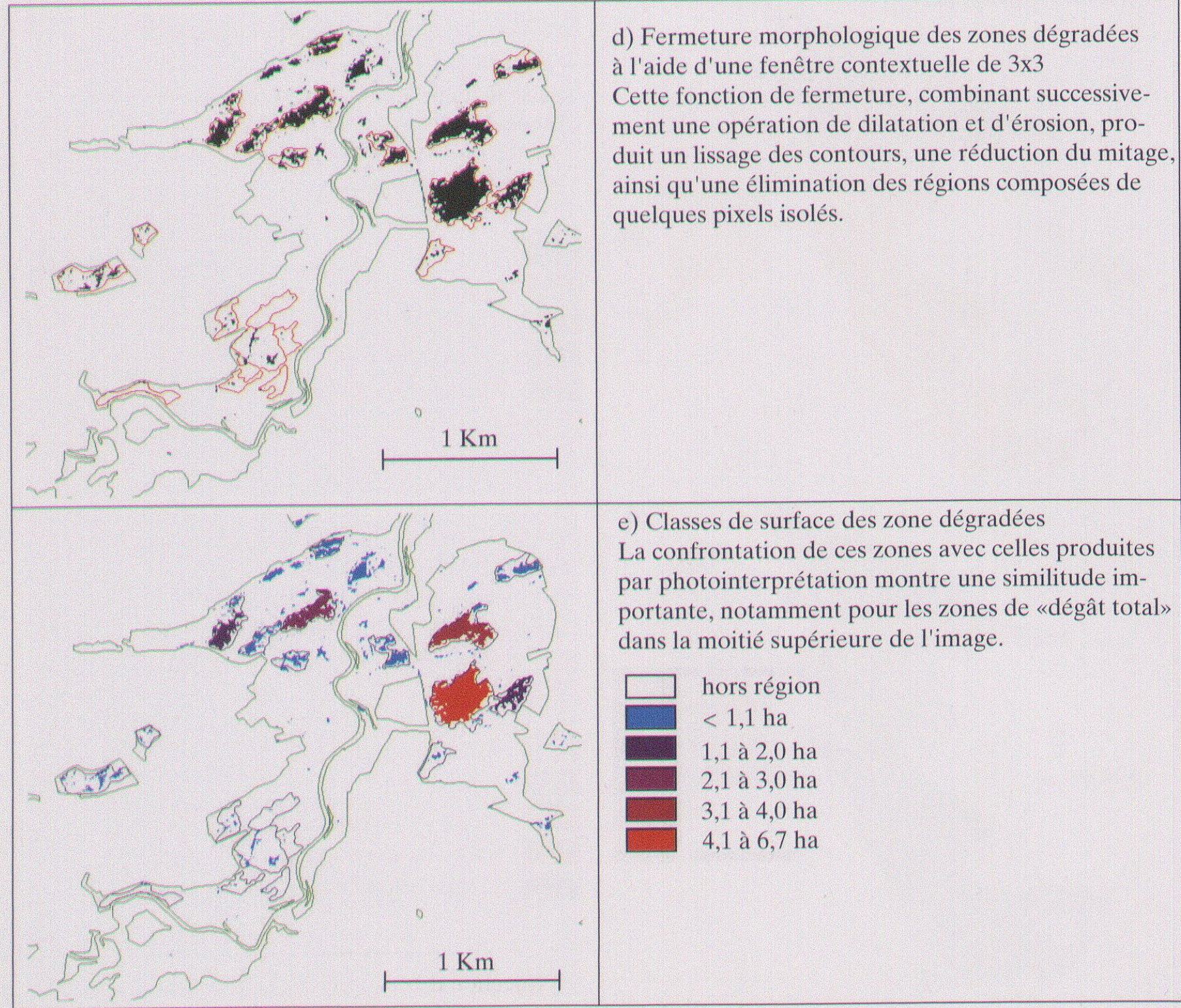

Figure 4: Résultats et commentaires des différentes étapes de la démarche de classification non dirigée. Comparaison avec les résultats obtenus par photointerprétation

Results and comments to the main steps of the clustering process and comparison with those obtained by photointerpretation

Resultate und Kommentare zu den einzelnen Schritten der unüberwachten Klassifikation. Vergleich mit den Resultaten aus der Photointerpretation

Source: Institut de Géographie de l'Université de Fribourg, Laboratoire de systèmes d'information - EPFL

paraître superflue. Il préfère probablement un zonage moins précis mais plus communicatif d'un point de vue visuel. Une substitution par une solution numérique suppose de définir des règles compatibles avec la logique de cette dernière et significative pour les décisions à prendre pour la gestion forestière. Ce travail de généralisation des contours reste à effectuer.

Analyse comparée des deux types de classification: Les deux processus de classification ont permis la détermination des zones forestières ouvertes, mais elles sont incapables de distinguer les clairières issues de l'ouragan Lothar et celles créées par l'homme. Seule une interprétation visuelle nous paraît susceptible de le réaliser. La classification produite par le logiciel eCognition s'est montrée performante pour la détermination des clairières toutes catégories confondues, confirmant ainsi le travail déjà cité de Schwarz et al. (2002). Ces deux observations suggèrent qu'une solution efficace passe par un processus mixte, alliant 


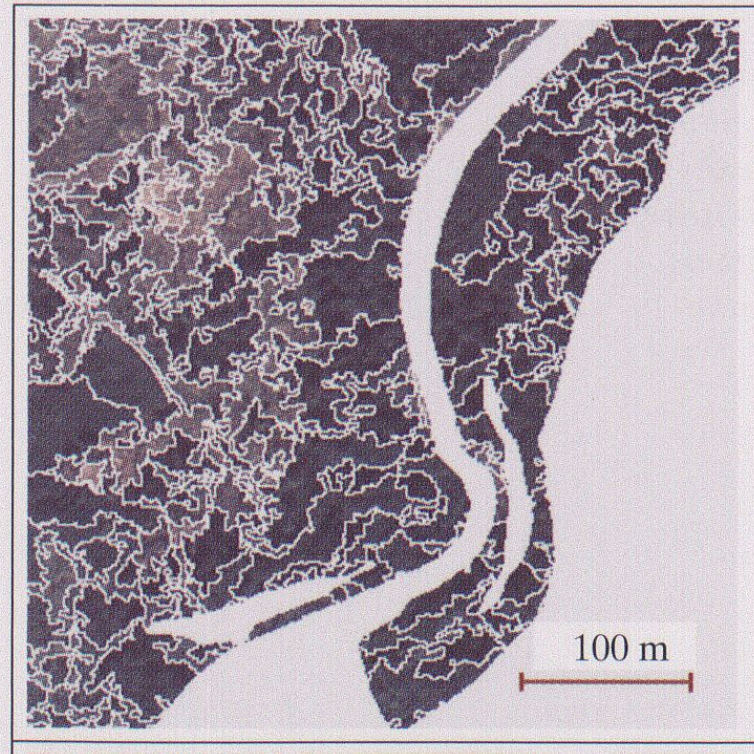

a) Première segmentation de l'image Les régions sont de petites tailles, spectralement homogènes. L'analyste dispose d'une première vision de la capacité discriminatoire offerte par l'image.

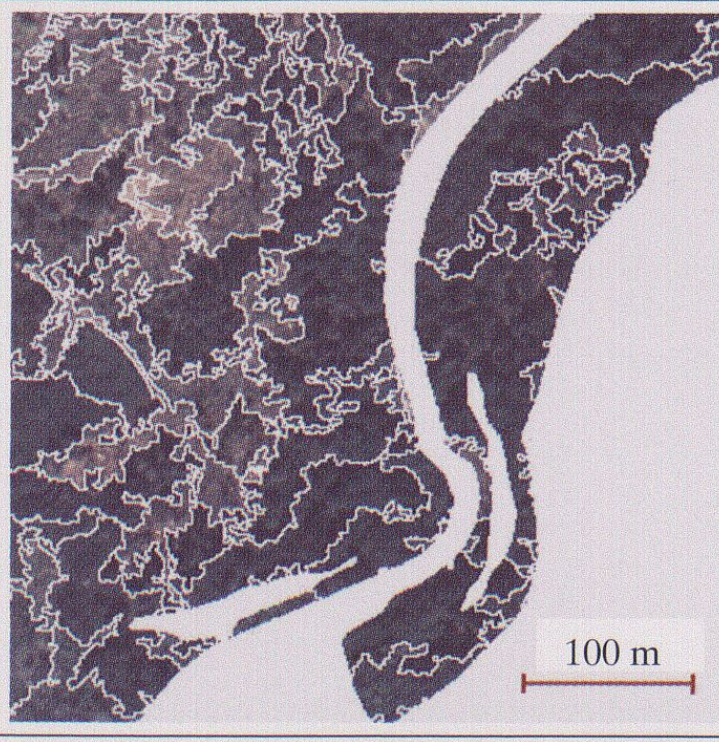

b) Croissane des régions de niveau supérieur

L'analyste interrompt la croissance des régions, lorsqu'il juge que les unités spatiales permettent une interprétation thématique suffisante pour sélectionner les plus significatives. Elles servent de signatures pour l'affectation des autres unités (image 4c).

Paramètres eCognition introduits à ce stade: Spectral: 0.8, Spatial: 0.2, Rugosité: 0.5 . Compacité: 0.5, Seuil: 90

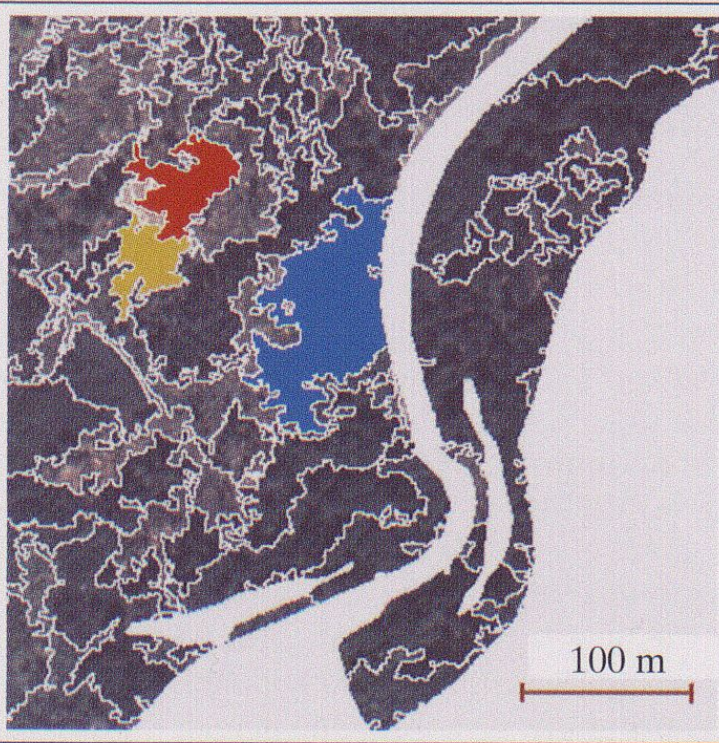

c) Injection des signatures

Par interprétation, l'analyste sélectionne les unités servant de référence à l'affectation des unités restantes à des catégories thématiques. 


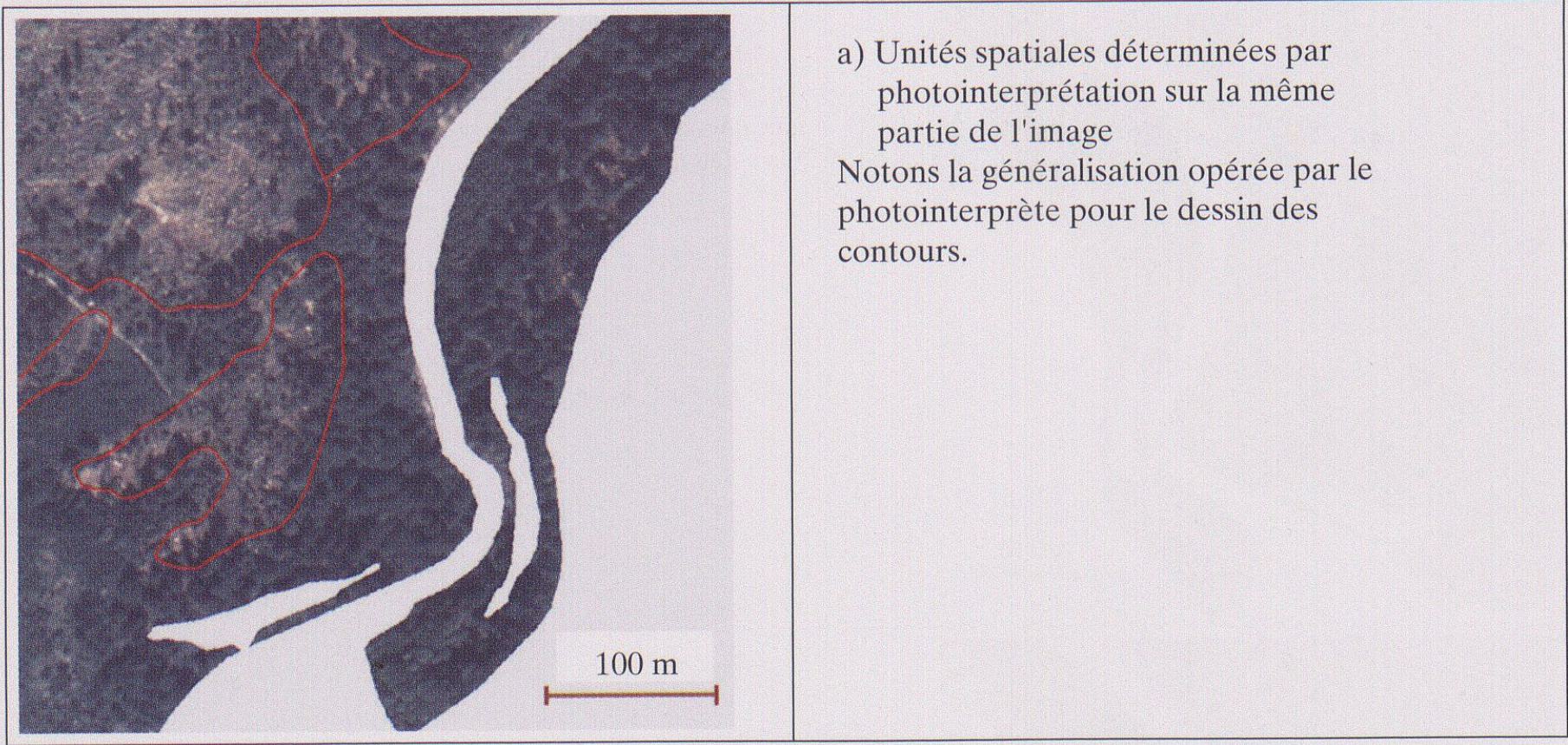

Figure 5: Processus de segmentation de l'image par croissance de région selon l'algorithme du logicial eCognition (illustration d'un extrait de la zone d'étude)

Image segmentation process in regional growth classification using eCognition software algorithms (illustrated by a study area subset)

Bildsegmentierung anhand der «regional growth»-Klassifikation mit der Computersoftware eCognition (Darstellung eines Ausschnittes des Studiengebietes)

Source: Laboratoire de systèmes d'information géographique - EPFL

le numérique et l'interprétation. L'approche par croissance de région laisse une grande part d'interprétation à l'analyste pour conduire son travail. Pour parvenir à des solutions opérationnelles, il conviendrait de procéder à d'autres expérimentations, en vue d'intégrer d'autres spécificités d'images et de situations forestières.

\section{Remerciements}

Nous remercions M. Vincent Grandgirard du Service des forêts du canton de Fribourg, d'avoir eu l'amabilité de nous fournir les images aériennes et les résultats de photointerprétation.

\section{Références bibliographiques}

Ahuja, N., An., B. \& B. Schachte (1985): Image representation using Voronoï tesselation. - Computer Vision Graphics, Image Processing (CVGIP) 29: 286-295.

Caloz, R. \& C. Collet (2001): Traitements numériques d'images de télédétection. - Presses de l'Université du Québec/AUPELF, Québec, 380 p.

Chassery, J.M. \& M. Melkemi (1991): Diagramme de Voronoï appliqué à la segmentation d'image et à la détection d'événements en imagerie multi-sources. Traitement du signal 8, 3:155-164.

Cocquerez, J.-P. et S. Phillip (1995): Analyse d'images : filtrage et segmentation. - Paris: Masson: $453 \mathrm{p}$.

ECognition (2001): eCognition - Object based image analysis, User Guide v 2.1, DEFINIENS AG - Rindermarkt 7 - D-80331 München, Germany.

HARALICK, R.M. \& L.G. SHAPIRO (1985). Survey: Image segmentation techniques, Computer Vision Graphics, Image Processing (CVGIP) 29: 100-132.

HojJatoleslami, S.A. \& J. Kittler (1998): Region growing : a new approach, IEEE transactions on Image processing 7, 7:1079-1084.

INAMPUDI, R. \& NMURALIKRISHNA (1999): Application of region growing algorithm for classification of IRS multispectral image. - IEEE: 1260-1282.

NAgaO, M. \& T. MatsuYama (1979): Edge preserving smoothing, Computer graphics and image processing 9, 4: 394-407.

SonKa, M., Hlavac, V. \& R. Boyle (1998): Image processing, Analysis and Machine vision. - London: PWS Publishing: $770 \mathrm{p}$.

Schwarz, M., Steinmeier, C. \& L. Waser (2002): Detection of storm losses in Alpine forest areas by different methodical approaches using high-resolution satellite 


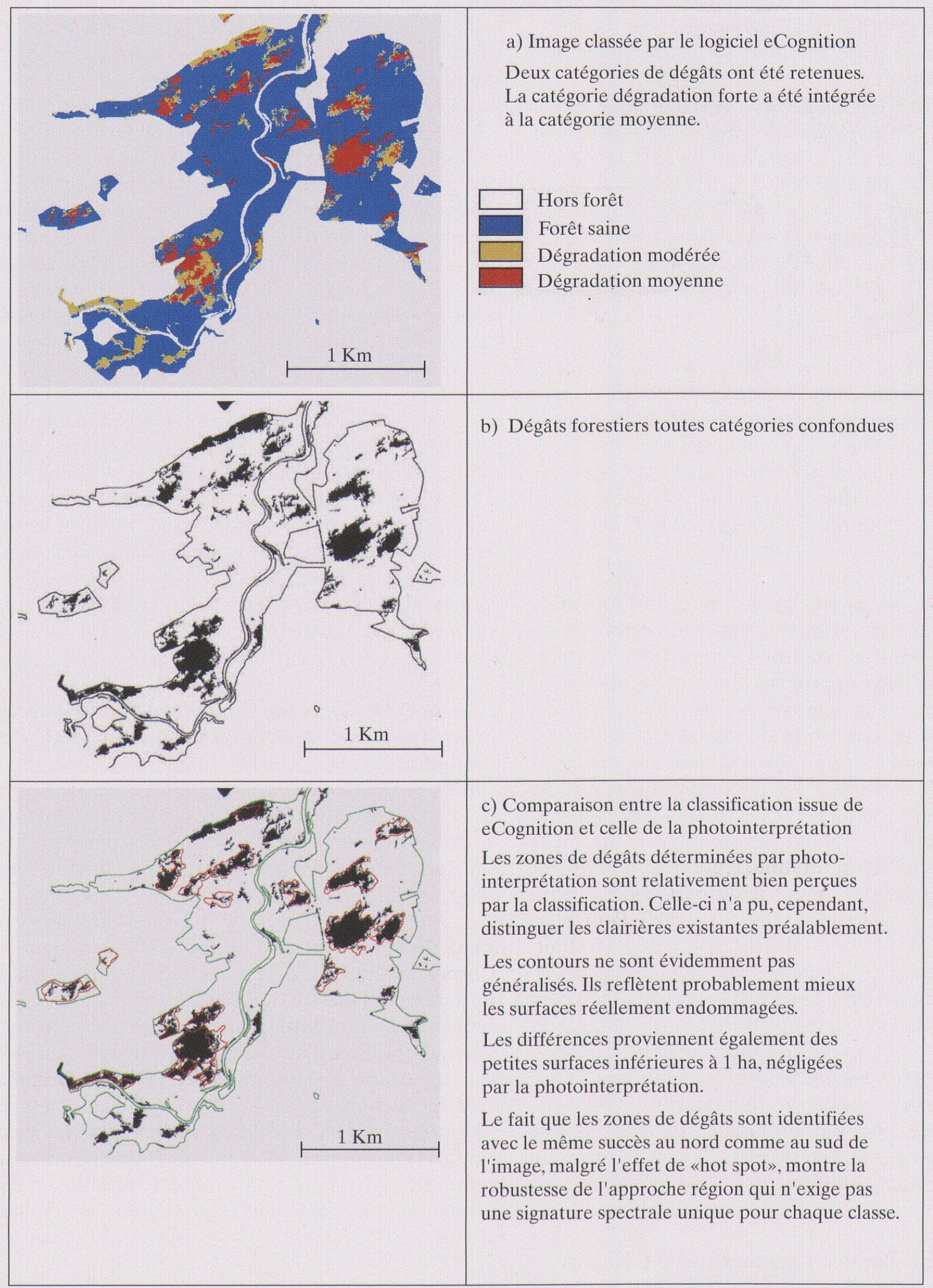

Figure 6: Résultats de la classification issue de eCognition et comparaison avec ceux obtenus par photointerprétation

Results and comments from main steps of the regional growth classification process and comparison with those obtained by photointerpretation

Vergleich der mit e Cognition erzielten Klassifikationsresultate mit jenjenigen der Photointerpretation Source: Laboratoire de systèmes d'information géographique - EPFL 
data. - In: BegNi, G. (ed.): Observing our environment from space. New solutions for a new millenium. - Lisse: Balkema: 251-257.

Vincent, L. et P. SoILle (1991): Watershed in digital espaces: An efficient algorithm based on immersion simulation. - In: IEEE Transactions on Pattern Analysis and Machine Intelligence 13, 6: 583-598.

YU, Y.-W. \& J.-H. WANG (1999): Image segmentation based region growing and edge detection. - International Conference on Systems, Man, and Cybernetics, 1999, IEEE Systems, Man, and Cybernetics '99, 6: 798-803.

\section{Résumé: Approche comparée de méthodes de classification d'images aériennes: une étude de cas} L'exploitation d'images aériennes ou satellitales à très haute résolution $(<5 \mathrm{~m})$ remet en cause des procédures de classification qui pourtant avaient fait leur preuve pour l'exploitation d'images à plus faible résolution.

Par une étude de cas portant sur les dégâts forestiers provoqués par le passage de l'ouragan Lothar en fin décembre 1999, nous mettons en évidence le comportement de deux approches, l'une conventionnelle basée sur une classification non-dirigée, l'autre exploitant la notion de croissance de région. Ces méthodes numériques sont mises en relation avec une cartographie des dégâts réalisée par photointerprétation. On montre que photointerprétation et approche numérique ne peuvent être comparées en termes strictement quantitatifs du fait de règles différentes intervenant dans la généralisation des contours des unités spatiales.

Un bref résumé des principes sur lesquels est construite la classification par croissance de région est présenté. L'image aérienne utilisée présentant un fort effet de «hot spot», il a été possible de mettre en évidence une plus grande robustesse des signatures définies par catégorie thématique dans le cas de la classification par croissance de région. Les zones de dégâts sont déterminées avec le même taux de réussite, indépendamment de leur position dans l'image.

\section{Summary: Comparative Approach to the Classifica- tion Methods of Aerial Images: a case study}

The current expanding availability of high resolution satellite and aerial images in digital form $(<5 \mathrm{~m})$ has forced image processing analysts and developers to reconsider classification strategy and procedures. Classical procedures, although improved and validated for low resolution images, are no longer able to make full use of the richer and more complex high resolution image content.
Using a case study on the evaluation of Lothar hurricane forest damages, the authors intend to compare the process and performances of two different classification approaches: a classical unsupervised method - clustering - and a regional growth classification method. Results are then compared with those obtained by photo-interpretation. Although results from a visual interpretation and from numerical processing cannot be compared on a quantitative basis, due to the use of different rules applied for contour generalisation of spatial units, it is meaningful to consider the results of photo-interpretation process as a reference for this comparison.

This paper includes a brief presentation of the regional growth classification procedure, emphasising basic principles and procedure steps. As the aerial image used in this experimentation contains a strong hotspot effect, it demonstrates the strength of thematic signature definition proposed by the regional growth method. All damaged areas were successfully depicted throughout the heterogeneously illuminated image with this latter method, as unsupervised classification produced less satisfying results in weakly reflective zones.

\section{Zusammenfassung: Eine vergleichende Annäherung an die Klassifikationsmethoden von Luftbildern: eine Fallstudie}

Für die Auswertung hochaufgelöster Luft- und Satellitenbilder (Maschenweite $<5 \mathrm{~m}$ ) müssen klassische Klassifikationsmethoden, die zwar bei einer geringeren Auflösung der Bilder gute Resultate erzielen, neu beurteilt werden.

Anhand einer Fallstudie über Waldschäden des Sturms Lothar werden zwei Ansätze untersucht, eine konventionelle unüberwachte Klassifikation und ein Verfahren, das auf dem regional growthKonzept beruht. Die Resultate werden dann mit denjenigen der Kartographie durch Fotointerpretation verglichen. Dabei stellt sich heraus, dass ein rein quantitativer Vergleich zwischen den beiden numerischen Methoden und der Fotointerpretation unmöglich ist, da die Konturen der räumlichen Einheiten anhand verschiedener Regeln generalisiert werden.

Die Grundlagen der auf dem regional growth-Konzept basierenden Klassifikationsmethode werden kurz erläutert. Dank der Präsenz eines starken hot spotEffektes im verwendeten Luftbild konnte gezeigt werden, dass die regional growth-Methode robustere Signaturen für die thematischen Klassen generiert. Unabhängig von der Position im Bild, werden die Schadenflächen mit derselben Erfolgsquote erkannt und ausgewiesen. 


\section{Orientation pédagogique}

- Comment estimer les dégâts forestiers produits par l'ouragan Lothar?

- Quelle procédure choisir pour une classification d'image à très haute résolution?

- Quelles sont des caractéristiques d'une classification par «croissance de région»?

- Peut-on évaluer de la même manière une segmentation d'image issue d'une photointerprétation et celle produite par une classification numérique?

Phys. Dip. Régis Caloz et Ing. Dip. Abram Pointet, Faculté de l'Environnement naturel, architectural et construit, Laboratoire de Systèmes d'information géographique, Ecole polytechnique fédérale de Lausanne, CH-1015 Lausanne.

e-mail:

regis.caloz@epfl.ch

abram.pointet@epfl.ch

Prof. Dr. Claude Collet, Departement de Géosciences, Institut de Géographie, Université de Fribourg, Pérolles, $\mathrm{CH}-1700$ Fribourg.

e-mail: claude.collet@unifr.ch

\section{Manuskripteingang/received/manuscrit entré le} 17.2.2003

Annahme zum Druck, accepted for publication, accepté pour l'impression: 5.6 .2003 